\title{
Propuesta de coordinación de servicios de orientación educativa: fortaleciendo la calidad en educación a distancia
}

Ligia Arguedas Ramírez

Programa de Orientación y Atención Psicoeducativa, Universidad Estatal a Distancia, (UNED). Costa Rica; larguedas@uned.ac.cr

Recibido: 24 de junio del 2015
Aceptado: 03 de agosto del 2015

\section{Resumen}

En este artículo se plantean aspectos generales que fundamentan el propósito del Programa de Orientación y Atención Psicoeducativa -POAP-, su gestión de apoyo y servicios de orientación educativa a las estudiantes y los estudiantes de la Universidad Estatal a Distancia y; marco de referencia a la propuesta de coordinación formal e institucionalizada con la academia: escuelas, programas de estudio y cátedras. Una propuesta, cuyo propósito es colaborar y favorecer de manera sistémica y transversal a un quehacer académico con calidad en el sistema de educación superior a distancia. Se propone concretar la coordinación en procura de potenciar y fortalecer el acompañamiento académico, personal, social, vocacional y ocupacional de la población estudiantil; mediante un impacto positivo en su rendimiento académico y la conclusión exitosa y en tiempos oportunos de su proyecto de estudio en la Universidad, como indicadores de calidad institucional.

Palabras clave: orientación educativa, calidad, prevención, desarrollo, formación, participación e integración.

\footnotetext{
Abstract

Service coordination proposed educational guidance: strengthening the quality in distance education

This article raises general issues underlying the purpose of the Program in Educational Counseling and Assistance -POAP- and management support services and educational guidance to students of the University and, under reference to the proposal for formal coordination and institutionalized academia: Schools, Curriculum and Chairs. Proposals whose
}

main purpose is to collaborate and promote systemic and transversely to a quality academic work in the system of higher distance education. It is proposed to realize this coordination; in an attempt to enhance and strengthen the academic, personal, social, vocational and occupational accompaniment of the student population; with a positive impact on their academic performance and the successful and timely conclusion times their research project at the University, as indicators of institutional quality.

Key words: educational counseling, quality, prevention, development, training, participation, integration.

\section{INTRODUCCIÓN}

El Programa de Orientación y Atención Psicoeducativa-POAP, de la UNED, se concibe como un proceso educativo sustentado en el humanismo, en el constructivismo y las teorías de desarrollo humano, en el contexto de la educación a distancia; así como en la misión y visión de la Universidad Estatal a Distancia-UNED-de democratizar y dar acceso a la educación superior.

EI POAP ofrece servicios orientativos y psicoeducativos a la población estudiantil de la Universidad, con el propósito de favorecer su crecimiento académico, socio-afectivo, vocacional y ocupacional, lo cual resulta 
en el logro académico y el desarrollo de sus habilidades y potencialidades personales y cognitivas. Se propone como apoyo transversal al quehacer académico de la Universidad: escuelas, programas de estudio, cátedras y centros universitarios; con una línea de acción investigativa, teórica, práctica y propositiva.

También articula desde sus inicios, en el año 1994, la prestación de los servicios desde el área de vida estudiantil de la Universidad, enfocando dos aspectos fundamentales: las estudiantes y los estudiantes y su relación positiva y de logro de las metas de estudio en el sistema universitario a distancia (Arguedas, 2003).

En esa dirección y ampliando los planteamientos de Rodríguez, citado por Bisquerra (1992) y dimensionados en el modelo a distancia; el accionar del Programa se guía por tres principios fundamentales de la orientación como proceso educativo:

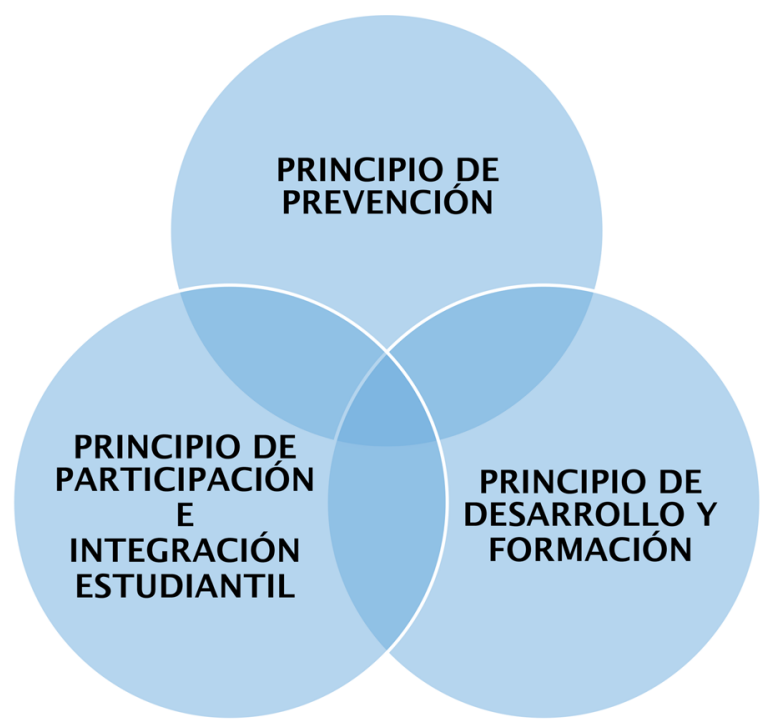

Figura 1. La orientación como proceso educativo . Fuente: POAP-UNED, Arguedas (2015)

\section{Principio de prevención}

Refiere a una actuación orientadora, un acompañamiento propositivo y proactivo hacia el estudiantado para fortalecer aspectos personales y de su dinámica de estudio y aprendizaje, los cuales permitan anticipar un buen manejo en su proceso de incorporación y permanencia en la Universidad.
Es decir, brindarle oportunidades y posibilidades que le comprometan y motiven con sus estudios universitarios.

\section{- Principio de desarrollo y formación}

Estrategias que promueven el desarrollo académico y el crecimiento personal de la población estudiantil en todas las áreas y mejoran su calidad de vida.Además, de enfatizar en que la universidad les brinda una preparación profesional, pero también y de manera esencial, una educación y formación "para la vida". Prevalece la concepción que en cualquiera de los programas de estudio y nivel (pregrado, grado y posgrado) la preparación universitaria implica principios formativos integrales $y$ fundamentales de convivencia humana y compromiso social, en un entorno diverso y multicultural.

\section{- Principio de participación e integración estudiantil}

La participación e integración estudiantil destacan, de manera importante, como procesos vivenciales que permiten que las estudiantes y los estudiantes desarrollen su sentido de pertenencia, identificación y compromiso con la Universidad y con las metas de estudio propuestas. En un modelo de educación a distancia la participación del estudiantado es imperativa; lo cual podría apreciarse como paradójico, pero es un aspecto trascendente. Se percibe como un elemento activo de involucramiento que lo integra en un "proceso educativo de aprendizaje", en el que pueden decidir, asociarse, opinar y transformarse; gestando a la vez motivación intrínseca, compromiso y responsabilidad para el logro de su proyecto de estudio en la UNED (Vam Dam, 1997, citado por Arguedas y Jiménez, 2004).

Estos principios del Programa se implementan con propuestas y estrategias de orientación vinculadas a la educación superior a distancia; como factor institucional determinante de éxito y buen desempeño académico de la comunidad estudiantil. Con una labor de acompañamiento para su desarrollo y que posibilite su adecuada y efectiva relación con las competencias, características y responsabilidades que este modelo de estudio implica. 
Como resultado de lo anterior, la Propuesta de coordinación de servicios de orientación educativa con la academia comprende una estructura que va desde su propósito, su fundamento en el contexto teórico-conceptual del POAP y la calidad universitaria; así como algunos principios clave para hacer efectiva esa coordinación; tanto de orden institucional como estudiantil, el cómo de su implementación y algunos de los principales servicios y procesos de orientación, que de manera inicial se desarrollarían en la propuesta.

\section{Propuesta de coordinación de servicios de orientación educativa con la academia-UNED: escuelas, programas de estudio, cátedras y centros universitarios}

\section{Propósito de la propuesta}

La experiencia del POAP con los servicios estudiantiles en los Centros Universitarios de la Universidad, algunos hallazgos en la evaluación de los diferentes proyectos y en resultados de investigaciones y estudios regulares que se efectúan en el Programa, respaldan el criterio de que el estudio a distancia requiere una mediación y orientación que apoye al estudiantado, le facilite un aprendizaje independiente, eficaz, significativo y le dirija a la autonomía y la autorregulación en sus estudios, como aporte sustantivo a la calidad académica y valor agregado de la preparación profesional.

Autores como Salmerón (2002) sustentan lo anterior con el criterio de que las universidades han de asumir un papel determinante en dos aspectos:

a. Como ámbito de reflexión intelectual, tiene la obligación y responsabilidad de liderar los cambios que conducen al progreso social y humano.

b. Como responsable de asegurar el nivel de preparación que exigen las sociedades modernas, debe promover los principios de equidad e igualdad para todos los que quieran acceder a la educación universitaria y desarrollarse a través de ella (Salmerón, 2002, p. 2).

Además de cubrir nuevas realidades y demandas que Salmerón también puntualiza; por ejemplo:
- Hacer compatible el desarrollo y la formación científica de la población estudiantil y del profesorado con el desarrollo de competencias personales, sociales y profesionales para la carrera; como proyecto vital y de inserción comprometida y satisfactoria al mundo laboral.

- Una planificación académica, "flexible y eficiente", que permita atender una población con perfiles de ingreso cada vez más variables, que apuntan a la diversidad; sin perder la calidad académica.

- Brindar una atención más personalizada al estudiantado mientras esté en la Universidad, así como a las graduadas y los graduados en su pronta incorporación laboral.

Los anteriores señalamientos contextualizados en los sistemas de educación a distancia, en los que la población estudiantil puede sentirse aislada y con poco sentido de pertenencia, demandan un accionar institucional de estrategias comunes, para todas y todos los involucrados en el proceso educativo, vinculadas con propósito y planificación intencionados. Según Borges (2005) se presentan implicaciones para la comunidad estudiantil, la docente y la institución en sistemas de estudio universitario de cursos en línea, pero con validez para sistemas abiertos y a distancia, ya que pueden generar frustración y desmotivación en las y los estudiantes que afecte negativamente sus estudios y aprendizaje.

- La propuesta en el contexto teórico y conceptual del Programa de Orientación y Atención Psicoeducativa-POAP

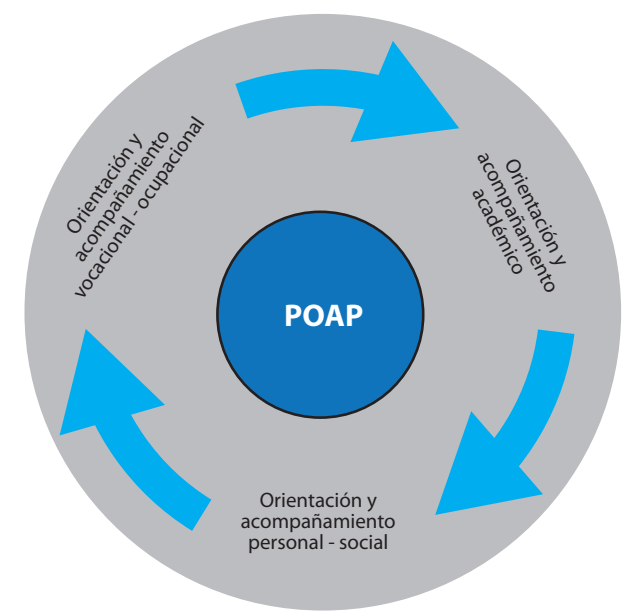

Figura 2. Ejes conceptuales del Programa de Orientación y Atención Psicoeducativa-POAP. Fuente: POAP-UNED, Arguedas (2015) 


\section{Orientación y acompañamiento académico}

Se propicia, como bien lo señala Montanero (1998) citado por Arguedas (2003), alcanzar uno de los principios de la orientación y la intervención psicopedagógica "personalizar la labor educativa":

Por un lado se trata de una actividad conscientemente dirigida a individualizar y adaptar el proceso de enseñanza - aprendizaje a las necesidades de cada alumno. Por otro, debe procurar garantizar que ese proceso no redunde sólo intelectualmente, sino en todo el desarrollo integral de su personalidad. (Montanero, 1998, citado por Arguedas, 2003, p. 149)

Sumado lo anterior al criterio de Hernández (2004) quien define la educación como formación de sentido "y, sobre todo, formación de un sentido personal, anticipación y acción meditada y responsable sobre el lugar y tareas del individuo en la sociedad y su autorrealización personal que debe contribuir, por lo tanto, a la formación coherente de la identidad personal y social plenas" (Hernández, 2004, p. 7).

Bajo esa línea de trabajo, en el POAP se diseñan procesos de inducción para estudiantes en el aprendizaje independiente como estrategia que, desde la conceptuación de la orientación educativa, permiten articular los valores institucionales con sus experiencias personales, la identificación y reflexión de sus motivaciones, su nivel de compromiso y sus necesidades de aprendizaje (Arguedas, 2003). Así como la reflexión sobre el modelo de educación superior a distancia; con información y generación de conocimiento en la población sobre sus características, programas y servicios que le faciliten su incorporación, adaptación y, por lo tanto, su mejor desempeño dentro del sistema (Arguedas, 2002). Respetando, por supuesto, los ámbitos de competencia institucional al atribuir que el rendimiento y las calificaciones obtenidas por cada estudiante en las asignaturas de su plan de estudio están referidas a la gestión de los procesos de enseñanza y aprendizaje -ejercicio directo de la academia-, la entrega de la docencia, la gesta pedagógica, la evaluación de los aprendizajes, el bagaje educativo del propio estudiante y del tutor o profesor, entre otros; sea en un sistema presencial o a distancia. Pero que, no obstante, conjunta e implica una variedad de factores y responsabilidades por parte del estudiantado y de la comunidad universitaria como un todo.

\section{Orientación y acompañamiento personal-social}

Integra todo lo relacionado con el desarrollo de habilidades sociales, el proyecto de vida, la educación emocional, la motivación, el bienestar, la calidad de vida $y$, en general, el desarrollo humano promovido desde el contexto universitario a distancia (Arguedas, 2004).

De acuerdo con lo anterior, y desde la perspectiva del Programa, el paso de las y los estudiantes por la Universidad debe trascender a la preparación académica con una formación:

(...) [que] genere cambios significativos y permanentes en su desarrollo personal, esperando que alcancen un crecimiento que se constate en su forma de percibir el mundo, de relacionarse consigo mismos y con los otros y, de alguna manera, de afrontar la vida con responsabilidad, tolerancia a la diversidad y como gestores y promotores de cambio y compromiso social (Arguedas, 2004, p. 1)

La práctica de los servicios de orientación, en este eje conceptual, brinda al estudiantado la oportunidad de analizar y reflexionar sobre su proceso de desarrollo como personas. Se busca que profundicen en su propio conocimiento y su relación y desenvolvimiento en el contexto de su realidad social.

Los proyectos atendidos utilizan una metodología participativa y vivencial de trabajo en grupo; principalmente talleres con temáticas sobre autoestima, motivación, manejo del estrés en los estudios a distancia, sexualidad, valores y competencias e inteligencia emocional, con iniciativas en el campo de la promoción de la salud, entre otros (Arguedas, 2003).

\section{Orientación y acompañamiento vocacional-ocupacional}

La complejidad del mundo laboral en los tiempos actuales y la responsabilidad en la elección profesional como decisión que adscribe a una particular forma de vida, justifican y otorgan una relevancia trascendental a la intervención educativa en los procesos de orientación vocacional y ocupacional. El surgimiento continuo de nuevas profesiones y ocupaciones, la movilidad profesional y la "reconversión profesional" acrecientan la incertidumbre y dificultan los distintos instantes en que las personas deben elegir, ya sea su preparación o su especialización (Álvarez, M. y Bisquerra, R., 1991). 
En ese sentido, se orienta al educando en su desarrollo vocacional, entendiéndose:

(...) como un proceso inherente al desarrollo personal. Un proceso de vida que permite encontrar un norte, un lugar en el mundo laboral. Un lugar que puede ser fuente de bienestar y satisfacción, si es el resultado de un profundo conocimiento de sí mismo, las posibilidades y recursos propios y del contexto social y cultural del que se forma parte (Arguedas, 2002, p. 29).

El eje conceptual se concreta en el accionar del Programa con talleres vocacionales y ocupacionales y la atención individual de estudiantes. Se favorece el autoconocimiento, el conocimiento del medio y la toma de decisiones que les permite plantease un proyecto de formación profesional en la Universidad y la orientación necesaria en el ámbito ocupacional para su exitosa inserción en el mundo laboral (Arguedas, 2003).

\section{- La propuesta en el contexto de la calidad universitaria}

Unificar un concepto de calidad universitaria, según De Ketele (2008) citada por Boni y Gasper (2011), es difícil por su carácter multidimensional y relativo; además de concebirse como un proceso político en sí mismo. Tema también tratado por Apodaca y Lobato (1997) al afirmar que tanto la calidad como la pertinencia en la formación universitaria son exigencias que sobrepasan las fronteras institucionales demandas que pueden verse como "imposiciones" de agentes sociales, políticos y económicos externos. Por lo que igual de controversial es su evaluación y su gestión vinculadas a aspectos de poder y de valores - desafíos de la actividad académica- y que nunca, como ahora, ha estado tan cuestionada y en el ojo público (Boni y Gasper, 2011).

Lo anterior permite reflexionar sobre la responsabilidad que tenemos todos los que somos partícipes directos en el logro de una academia de calidad y, por lo tanto, de una formación universitaria con calidad. En donde las y los estudiantes no son solo beneficiarios del "proceso educativo", sino, también, su resultado (Apodaca y Lobato, 1997).

Ciertamente la UNED ha logrado por medio de sus Centros Universitarios en todo el territorio nacional posesionarse como una institución pionera en educación superior a distancia y como un medio de inclusión y movilidad social, con aportes concretos y exitosos.

Sin embargo, la tarea continúa con el apoyo institucional a diferentes prácticas y propósitos que permitan afrontar nuevos desafíos en la educación superior y se relacionen con la diversidad de la población estudiantil, un mundo laboral cambiante y competitivo, el tipo de institución, su misión y, por consiguiente, con una mayor exigencia y resultados del estudiantado.

En ese marco, el concepto de calidad supone una visión de sistema integrado, innovación e interdisciplinariedad. La calidad "como derecho de las personas estudiantes" a quienes se les exige una óptima práctica profesional, resultante imperativo de una buena práctica educativa.

Esa es la dirección que fundamenta la propuesta: partir de que solo cuando logremos articular e integrar de manera sistémica todo el quehacer institucional como un proceso esencial y con una meta común las y los estudiantes y por medio de ellos la sociedad costarricense, estaremos orientándonos hacia una formación universitaria de calidad y pertinencia.

\section{- Principios básicos de coordinación}

Este tipo de coordinación entre dependencias de una misma universidad, su diseño, implementación y evaluación implican, como punto de partida (Alonso, Calles y Sánchez, 2012) el reconocimiento de las características institucionales y la identificación de algunos principios básicos que contextualizados en el ámbito universitario y en un sistema a distancia son de referencia clave para el éxito del proyecto. 


\section{Institucionales}

Creer en la importancia del estudiantado como centro del quehacer institucional.

Búsqueda de la excelencia en los servicios y atención a las y los estudiantes.

Una institución que logra que la población estudiantil desarrolle sentido de pertenencia, que se identifique con los principios y valores institucionales (su misión y visión). Que se apropie y se sienta parte de una comunidad educativa en un sistema de educación superior a distancia.

Una institución que brinda las condiciones académicas y favorece la concreción del proyecto de estudio claramente orientado al logro de metas y preparación profesional de la colectividad estudiantil.

Una institución en la que los procesos de acreditación de carreras y programas, por parte de las agencias nacionales e internacionales, reciben una colaboración formal y estructurada sobre los procesos de orientación y acompañamiento brindados a las estudiantes y los estudiantes en cada escuela y programa de estudio.

\section{Estudiantiles}

Participación voluntaria del estudiantado en los procesos de coordinación y servicios de orientación y psicoeducativos con las escuelas y los programas de estudio. Estimulando el compromiso y la responsabilidad de aportación activa de "fuerte voluntad" en sus estudios.

Desarrollo de una relación de confianza mutua de credibilidad, de la población estudiantil por la institución; su calidad y compromiso con su formación universitaria. Y de la institución en las posibilidades de cada estudiante, favoreciéndoles oportunidades para el logro y éxito en sus estudios, con procesos de enseñanza-aprendizaje de responsabilidad compartida.

Los servicios de orientación y acompañamiento académico que se coordinen con las escuelas, programas y cátedras dirijan al estudiantado al compromiso de seguir adelante de manera autónoma, independiente y auto gestionando su aprendizaje. Sin invalidarlos o subestimarlos, más como estímulo "que incite a la reflexión, la toma de decisiones y la actuación (...) del estudiante (...) por sí mismo" (Alonso, Calles y Sánchez, 2012, p.34).

Identificar y tratar de acompañar, en la medida de lo posible, las necesidades académicas de la persona estudiante, pero también teniendo en cuenta su emotividad, sus preocupaciones, sus temores y sus motivaciones.

Validar el sistema de estudio a distancia como un reto, una oportunidad en la que debe apropiarse de sus ventajas.

Facilitar la socialización y relación del estudiantado con sus compañeros, sus iguales, aunque el sistema sea a distancia: que no se sienta solo ni aislado pues cuenta con un conjunto de apoyos académicos que le colaboran en sus procesos de estudio.

\section{- El cómo de la propuesta}

- La Oficina de Orientación y Desarrollo EstudiantilOFODE, en conjunto con la Vicerrectoría Académica, designarán a un profesional de Orientación Educativa del POAP en cada una de las escuelas, por acuerdo y períodos específicos.
Este será el vínculo oficial entre las dependencias y el responsable de articular y mediar los diferentes servicios de orientación. También, participará de los consejos de escuela, como invitado permanente, con voz aunque no con voto.

- En cada escuela se creará un equipo de orientación y guía académica conformada por los 
coordinadores/as de carrera y el orientador/a designado.

- $\quad$ Este equipo será dirigido, siempre y por períodos específicos, por alguna de las y los académicos coordinadores de carrera participantes.

- Las responsabilidades y tareas de estos equipos de orientación y guía académica de las escuelas, serán las de identificar servicios y procesos específicos de orientación en sus poblaciones estudiantiles, de apoyo académico y vínculo con otras oficinas y los diferentes programas de la Oficina de Orientación y Desarrollo Estudiantil y de la Dirección de Asuntos Estudiantiles-DAES (Registro, Atención Socioeconómica, Promoción Estudiantil, poblaciones específicas: con discapacidad, privados de libertad, en el exterior y de movilidad estudiantil, entre otros), la Defensoría de los Estudiantes, la Federación, las asociaciones estudiantiles, la Dirección de Centros Universitarios, instancias todas de la estructura organizacional de la UNED.

- El o la orientadora designada en cada escuela deberá gestionar y liderar procesos y servicios de orientación educativa para toda la población estudiantil de la Universidad, con fundamento en el modelo de educación a distancia y en los principales ejes de la orientación como proceso educativo: el desarrollo académico, personal y social, vocacional y ocupacional.

- La persona designada promoverá, en cada escuela, servicios de orientación educativa que favorezcan la inducción, la incorporación la adaptación y la permanencia exitosa de todas y todos los estudiantes de la Universidad, en los centros universitarios.

- $\quad$ Será responsable de promover y vincular los servicios, procesos y proyectos de orientación, del POAP y la Oficina de Orientación y Desarrollo Estudiantil, en los centros universitarios de la Universidad; con criterios del equipo de orientación y guía académica de cada escuela.

- Coordinará, asesorará y formalizará la articulación de los diferentes servicios de orientación educativa con la Vicerrectoría Académica; las escuelas, los programas de estudio, las cátedras, instancias de la Vicerrectoría de Investigación, la Dirección de Producción de Materiales Didácticos, el Área Curricular, la Editorial, oficinas de la DAES y otras dependencias institucionales relacionadas con los servicios de orientación al estudiantado, en procura de su desarrollo y logro académico.

- Agenciará, de manera articulada, vínculos con instituciones y dependencias estratégicas para el buen desarrollo del Programa y los servicios al estudiante: (MEP, CONARE, universidades y otras).

- Coordinará, con el POAP y la OFODE, propuestas, seguimiento, designación de recursos (humano y material), diseños y rutas educativas de los diferentes servicios y procesos de orientación que se le brindará a los y las estudiantes, pero desde la detección de necesidades específicas por escuela y particularizando procesos de atención por carrera.

- Gestionará, con el POAP, la OFODE y equipo de orientación y guía académica de cada Escuela el establecimiento de normativa institucional que acuerpe y oficialice la propuesta de coordinación por medio del Reglamento de Gestión Académica y el Reglamento General Estudiantil de la Universidad, entre otros.

\section{Principales estrategias de gestión y servicios de orientación educativa del POAP que apoyarían la Propuesta de coordinación}

El propósito de los principales servicios de orientación estudiantil del POAP está claramente vinculado a la gestión y estructura de la Universidad y su modelo a distancia. Aunque algunos de esos procesos ya se desarrollan en el Programa; mantienen su robustez, vigencia y trascendencia como estrategia operativa para vincular y ejecutar la Propuesta. Desde sus comienzos -acuerdo del Consejo Universitario, sesión No. 1075-94, UNED-CR- se destaca la misión preventiva y de servicio del POAP al desarrollo integral de las y los estudiantes.

Así como brindar respuesta a una de las principales conclusiones del estudio de once centros universitarios de la UNED-CIEl-acuerdo 048-2001, UNED-CR, que señala como una de las posibles dificultades de la población estudiantil la percepción de "aislamiento" y de poca interacción que la Universidad establece con ellos. En este sentido, el POAP desarrolla diferentes procesos grupales de atención de estudiantes que permite 
su interacción y favorece su adaptación y permanencia en la Institución.

Por otra parte, llegar a la población estudiantil de manera más regional en los centros universitarios, en atención al acuerdo de desconcentrar servicios, incluidos todos los que se proporcionan en el área de Vida Estudiantil en los Centros Universitarios 100\% servicios. Propiciando, de esta forma, el desarrollo integral del estudiantado, así como de las diferentes regiones del país; según sesión 1499-2/2001 del Consejo Universitario, UNED-CR.
Además de concretar acciones pertinentes, necesarias y viables para la Universidad, en respuesta a lo dictaminado por el Consejo Universitario, UNED-CR, sobre admisión y matrícula (sesión 1630-2003, del 19 de marzo de 2003, Artículo 1, punto 4 de acuerdos), textualmente se indica:

Desarrollar programas de orientación para los estudiantes de primer ingreso, con la finalidad de propiciar su permanencia y éxito, de acuerdo con la capacidad institucional y prioritariamente en aquellos Centros Universitarios que muestran los mayores porcentajes de deserción.

CUADRO 2

Principales estrategias de gestión y servicios de orientación educativa del POAP

\section{Servicio}

Puestos de orientación en períodos de matrícula.

Citas de orientación e inducción académica. vocacional

Sesiones de orientación académica y estrategias de estudio a distancia

Sesiones de orientación personal - social

Citas de orientación y seguimiento académico.

\section{Descripción}

El proceso se realiza en los centros universitarios con servicios de orientación, todos los cuatrimestres.

Se llevan a cabo al inicio de cada cuatrimestre, la semana siguiente a la matrícula, en los centros universitarios. Las citas son sesiones grupales de orientación e inducción académica para estudiantes de primer ingreso. Se trabajan aspectos principales sobre el sistema a distancia, la entrega de la docencia, la organización académica y administrativa del centro universitario y de la Universidad. Así como los principales servicios de vida estudiantil y su acceso, el registro y la administración estudiantil, las becas de estudio, la promoción estudiantil (grupos de participación: artísticos, deportivos y voluntariado, entre otros); la Federación de Estudiantes y las asociaciones, la Defensoría de los Estudiantes, el Reglamento General Estudiantil: deberes derechos, gestión académica, entre otros.

Son sesiones grupales de orientación y seguimiento académico para estudiantes de primer ingreso. Se desarrollan a mediados de cada cuatrimestre en los centros universitarios con toda una estrategia de orientación y un tema de fondo (organización del tiempo de estudio, preparación para los exámenes, estilos de aprendizaje, entre otros). Es un espacio que permite mediar con las y los estudiantes, conocer cómo les va en los estudios, brindarles contención y apoyo, referirlos a las escuelas u a otros programas y servicios cuando corresponde.

Cada cuatrimestre en los centros universitarios se desarrolla un módulo educativo para el trabajo grupal en orientación vocacional, la escogencia de carrera y toma de decisiones, con cuatro sesiones de trabajo.

Es un módulo educativo para el trabajo grupal en orientación académica, con estrategias de estudio a distancia, herramientas para el estudio y el aprendizaje autónomo e independiente y procesos meta cognitivos.

Módulos educativos para el trabajo grupal en orientación personal -social. Se diseñan e implementan rutas educativas de intervención y acompañamiento en diversas temáticas: autoestima, sexualidad, habilidades sociales, motivación, manejo del estrés en los estudios y desarrollo personal, entre otros. 


\section{Servicio}

Sesiones de orientación ocupacional

Citas de orientación y acompañamiento académico

Procesos de orientación para estudiantes privados de libertad

Procesos de orientación para estudiantes con necesidades educativas especiales

\section{Descripción}

Módulo educativo para el trabajo grupal en orientación ocupacional y la incorporación al mundo laboral. Dirigido a estudiantes que están finalizando su carrera y prontos a incorporarse al mundo laboral.
Dirigido a estudiantes de la Escuela de Ciencias Exactas y Naturales-ECEN en asignaturas seleccionadas por alta reprobación y bajo rendimiento.

"Mi proyecto de estudio en la UNED y mis circunstancias personales" es coordinado con el Programa de Estudiantes Privados de Libertad, el POAP brinda un módulo educativo en orientación con temáticas de desarrollo personal en centros de atención institucional; por ejemplo, el Buen Pastor, la Reforma, San Rafael.

"Mi proyecto de estudio en la UNED desde mi vocación y mi realidad personal", es coordinado con el Programa de Estudiantes con Necesidades Educativas Especiales, el POAP brinda un Módulo Educativo en Orientación Vocacional autoconocimiento y realidad personal en al menos ocho regiones del país. Es una población que presenta alguna discapacidad, por lo que se atienden algunas temáticas que los motiven a seguir adelante en la consecución de su proyecto de estudio.

Es un proyecto institucional, dirigido a estudiantes indígenas que estudian en la Universidad. Se ofrecen servicios de incorporación y adaptación a la vida universitaria, desde su contexto, resguardo y evidencia de su cultura.

Con rutas educativas en diferentes temáticas y de acuerdo con necesidades específicas de las y los estudiantes, desde el ámbito del liderazgo estudiantil.
Procesos de orientación y articulación de servicios estudiantiles con la federación de estudiantes de la Universidad

Procesos de orientación y representación institucional en equipos y comisiones de trabajo en el ámbito universitario estatal

Proyecto de orientación y acompañamiento académico para estudiantes con bajo rendimiento y alta reprobación en sus estudios. Vicerrectoría Académica

Proyecto de Investigación y Desarrollo Académico POAP - PROIFED. Vicerrectoría de Investigación

Proyecto de Investigación sobre el perfil académico del estudiante de primer ingreso POAP-CIEI. Vicerrectoría de Planificación
Desde el POAP y su gestión se participa en tres comisiones que se coordinan en el Consejo Nacional de Rectores. Mediante procesos dirigidos a las y los estudiantes de universidades estatales y en temáticas del ámbito de la orientación educativa:

Comisión de Orientación, Divulgación e Información de la Educación Superior, Comisión de Éxito Académico, Comisión de Universidades Promotoras de la Salud: ejes sexualidad y habilidades para la vida.

Proyecto que se está desarrollando con la Escuela de Ciencias Exactas y NaturalesECEN. En asignaturas específicas con planes remediales, tutorías y materiales.

Desarrollo de procesos de investigación educativa y aplicación de resultados, los cuales fundamentan los servicios de orientación, la producción de materiales didácticos para el quehacer del POAP y la vinculación y diseño de proyectos con otras instancias; de implicaciones académicas.

Es un estudio sobre el perfil y características académicas de la población de primer ingreso. Es de carácter institucional con aportes de la orientación educativa y de implicaciones académicas. 
Proyecto de Movilidad Estudiantil. Comisión Institucional de Movilidad. Dirección de Internacionalización y Cooperación

Proyectos y servicios virtuales de Orientación. Oficina de Videoconferencia.
De implicaciones y desarrollo académico del estudiantado. Aportes desde la orientación educativa.

\section{Reflexiones sobre lo que favorecería, en el contexto institucional, la puesta en marcha de la coordinación de servicios de orientación educativa}

- Planificar procesos conjuntos de orientación y acompañamiento a la población estudiantil acordes con las necesidades de las escuelas, los programas de estudio y las cátedras. En concordancia con lo que las y los estudiantes requieren, independientemente del centro universitario en el que estudian.

- Lograr un mayor impacto de los servicios de orientación y acompañamiento psicoeducativo que aportan valor a la formación del estudiantado.

- Redoblar y potenciar esfuerzos para mejorar el rendimiento académico, disminuir la repitencia en asignaturas específicas, implementar planes remediales por escuelas y disminuir los tiempos de permanencia y graduación.

- Identificar población en riesgo académico con probabilidades de deserción y desarrollar estrategias conjuntas de atención.

- Motivar al estudiantado favoreciendo su incorporación, permanencia y logro académico.

- Atender necesidades reales de la población estudiantil.

- Impactar en la acreditación de programas de estudio, la regionalización de servicios, la equidad y el acceso a estos $y$, fundamentalmente, en resultados positivos en los índices de permanencia y rendimiento académico (Arguedas, 2010, p. 01).

- Lograr una "gestión transversal, multidisciplinaria y vinculante al quehacer académico de la Universidad" (Arguedas, 2010, p. 01).

- Aprovechar los recursos institucionales en el desarrollo conjunto de procesos, desde el aporte de las dependencias que se vincularían. Los recursos profesionales y económicos en los centros universitarios de la UNED en todo el país.

- Fortalecer el impacto y valor de los servicios de orientación educativa, sus alcances en el contexto institucional y la innovación en el acompañamiento académico estudiantil en modelos de educación a distancia.

\section{Síntesis y reflexiones finales}

- Integrar y articular las distintas acciones del Programa de Orientación y Atención Psicoeducativa-POAP en el proyecto educativo de la Universidad como un proceso inherente y de efecto transversal a su quehacer. Principalmente en los primeros niveles de formación donde se detecta los mayores grados de deserción y fracaso académico. Y como proceso estratégico en la calidad de la formación del estudiantado (Arguedas, 2003).

- Partir de que para la permanencia, la adaptación y el éxito académico de las y los estudiantes en la Universidad, lo motivacional y la capacidad o 
inteligencia emocional (lo afectivo, lo volitivo y lo social) son tan importantes como los aspectos intelectuales y racionales. Por tanto la necesidad de implementar servicios de orientación educativa que acompañen los procesos de estudio y aprendizaje del estudiantado con la atención de ejes transversales que favorezcan una formación integral (Arguedas, 2004).

- Fortalecer, mejorar y generalizar los procesos educativos que el Programa brinda al estudiante en el quehacer académico de la Universidad. Estas estrategias deben planificarse en el contexto institucional como responsabilidad compartida, con aportes de la docencia y sus requerimientos para un mejor desempeño del estudiantado; así como de la investigación para detectar las necesidades de estos, evaluar, mejorar los procesos y brindar seguimiento (Arguedas, 2004).

- El POAP cuenta con varios módulos educativos de orientación con diferentes estrategias de intervención grupal para la comunidad estudiantil. Son procesos motivacionales, de desarrollo personal y de inducción de las y los educandos en el aprendizaje independiente y en habilidades y estrategias de estudio, estilos de aprendizaje, comprensión de lectura, niveles de pensamiento, distribución efectiva del tiempo y otros aspectos del desempeño intelectual. Por su pertinencia y aporte en la formación estudiantil y el mejoramiento de la calidad académica deben desarrollarse en todos los centros universitarios con una cobertura total de la población y de forma coordinada con las escuelas. De manera se identifiquen con la Universidad y como estudiantes de esta modalidad educativa.

\section{REFERENCIAS}

Alonso, M., Calles, A. \& Sánchez, C. (2012). Diseño y Desarrollo de Programas de Mentoring. Equipo de Gestión ALFA GUÍA. Madrid: Universidad Politécnica de Madrid.

Álvarez, M. \& Bisquerra, R. (1991). Orientación Psicopedagógica. Material mimeografiado. España: Universidad de Barcelona.
Apodaca, P. \& Lobato, C. (1997). Calidad en la Universidad: Orientación y Evaluación. Barcelona: Editorial Alertes.

Arguedas, L. \& Jiménez, F. (1999). El Estudiante de la Educación a Distancia en la Perspectiva de un Nuevo Mileni. Programa de Orientación Estudiantil en la Universidad Estatal a Distancia de Costa Rica. Memoria. Congreso Internacional: San José, Costa Rica.

Arguedas, L. \& Jiménez, F. (2004). Las Citas de Orientación en un sistema de educación a distancia. Memoria. Primera Jornada de Intercambio de Experiencias Innovadoras en la Educación Universitaria. CONARE, Costa Rica.

Arguedas, L. (2002). Los Talleres de Técnicas de Estudio y Comprensión de Lectura: Una Experiencia Concreta en el Modelo de Educación a Distancia. Memoria Congreso Internacional sobre Tecnología y Educación a Distancia. Liderazgo en la Educación a Distancia con Excelencia y Exigencia Académica. Costa Rica.

Arguedas, L. (2002). Reflexiones sobre el desarrollo vocacional: una experiencia concreta. Innovaciones Educativas, VIII(13), 47-54.

Arguedas, L. (2003). La inducción del estudiante de primer ingreso en el aprendizaje independiente como requerimiento fundamental de la educación superior a distancia: Algunos resultados. X Encuentro Iberoamericano de Educación Superior a Distancia. Costa Rica.

Arguedas, L. (2004). Potenciación de la resiliencia: Una alternativa para la permanencia éxito académico de las y los estudiantes en la UNED. XII Congreso Internacional de Tecnología y Educación a Distancia.

Arguedas, L. (2010). Propuesta del Área de Orientación y Desarrollo Estudiantil. Dirección de Asuntos Estudiantiles. Material Mimeografiado. UNED: Costa Rica.

Bisquerra, R. (1992). Orientación psicopedagógica para la prevención y el desarrollo. Barcelona: Editorial Boixareu Universitaria.

Boni, A. \& Gasper, D. (2011). La universidad como debiera ser. Propuestas desde el desarrollo humano sostenible para repensar la calidad de la universidad, Sistema, 220-221, 99-115.

Borges, F. (2005). La frustración del estudiante en línea. Causas y acciones preventivas. Digithum, 7(7), 1-8. Recuperado de: http://www.uoc.edu/digithum/7/dt/esp/borges.pdf

Hernández, O. (2004). Proyecto de vida como categoría básica de interpretación de la identidad individual y social. 
Biblioteca Virtual. Consejo Latinoamericano de Ciencias Sociales. CLACSO. Buenos Aires, Argentina.

Mora, D. (2003). Educación de Adultos y Didáctica Universitaria. Centro de Capacitación en Educación a Distancia. Material Mimeografiado. UNED: San José, Costa Rica.
Salmerón, H. (2001). Los servicios de Orientación en la Universidad. Procesos y desarrollo. Ágora digital, 2. Recuperado de http://www.uhu.esp/agora/version01/ digital/numeros02/02-articulos/monografico/salmeron. htm

Universidad Estatal a Distancia. (2003). Vicerrectoría Académica. Modelo Pedagógico. Material Mimeografiado - borrador. UNED: San José, Costa Rica. 